\title{
Detection of Lung Nodules on Medical Images by the Use of Fractal Segmentation
}

\author{
Afsaneh Abdollahzadeh Rezaie, Ali Habiboghli* \\ Department of Computer Science and Engineering, Khoy Branch, Islamic Azad University, Khoy (Iran) \\ Received 2 September 2016 | Accepted 1 November 2016 | Published 23 December 2016
}

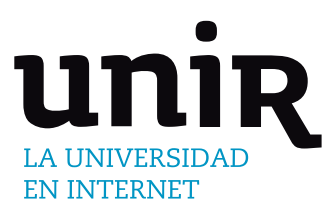

In the present paper, a method for the detection of malignant and benign tumors on the CT scan images has been proposed. In the proposed method, firstly the area of interest in which the tumor may exist is selected on the original image and by the use of image segmentation and determination of the image's threshold limit, the tumor's area is specified and then edge detection filters are used for detection of the tumor's edge. After detection of area and by calculating the fractal dimensions with less percent of errors and better resolution, the areas where contain the tumor are determined. The images used in the proposed method have been extracted from cancer imaging archive database that is made available for public. Compared to other methods, our proposed method recognizes successfully benign and malignant tumors in all cases that have been clinically approved and belong to the database.

\section{INTRODUCTION}

$\mathrm{M}$ ORE than 9 million people die annually around the world as the result of the chest diseases. Lung cancer is the second leading reason of death caused by cancer in the world [1]. More than $85 \%$ of all lung cancer cases have been attributed directly to smoking [2]. The reduction in mortality of lung cancer can be achieved by doing successfully the three strategies of quitting smoking, early detection and development of treatment methods [3]. At present, it is impossible to detect signs of lung cancer in the early stages, because they are similar to other diseases. Therefore, patients with lung cancer are usually detected in advanced stages of disease in which the treatment options are limited and this leads to a very low chance to survive. If patients are diagnosed in the earliest stages, the survival rate will be nearly $70 \%$. When patients are diagnosed at the stages of two and three, the survival rate drops dramatically to $34 \%$ and $13 \%$. The initial symptoms of the cancer can be coughing and hemoptysis which turn into chronic and recurrent infection of the chest [4].

Today, some of the common methods of biochemistry and pathology and graphic methods, including sputum cytology, chest radiography, fluorescence bronchoscope, polymerase chain reaction (PCR), computerized tomography (CT), low-dose computed CT scan and bronchial sampling are used widely to diagnose lung cancer [4]. For early detection of lung cancer, screening programs are applied to asymptomatic high-risk groups by several technologies such as sputum sampling, circulation of tumor biomarkers, serum proteome patterns, chest CT scan, nuclear magnetic resonance (NMR), or a combination of them (e.g., Chest X-rays by low-dose computed tomography (CT)), and other methods that have been the subject of study and debate over

* Corresponding author.

E-mail addresses: afsaneh.abdollahzadeh@yahoo.com (Afsaneh Abdollahzadeh Rezaie), habiboghli@iaukhoy.ac.ir(AliHabiboghli). the past few decades [5].

CT imaging techniques are advanced and more sensitive in the detection of lung cancer and have a high quality contrast. They are one of the best techniques for imaging soft tissue behind the bone structure. Modern CT machines enable fast acquisition of an accurate collection of successive images with very high resolution and support reliable detection. It been has proved that screening by CT scan has a strong potential to improve the likelihood of detecting lung cancer in its early and curable stages [6]. Computer-aided diagnosis (CAD) has been examined for the detection of nodules, because the computer can improve diagnostic accuracy [1,7]. For quantitative evaluation of results obtained from the analysis by computer systems, the physical measure of fractal dimension (FD) is used. The ability to determine ripples causes that the analysis of FD is especially useful in applications related to the tissues. Various amounts of FD show the structure of different tissues in the image. When the structure of the tissue is more complex, the amount of its FD will usually be higher [8].

Following section of this paper has been organized as follows: section 2 involves a literature review and an overview of the diagnosis of lung cancer by different methods. Section 3 describes the proposed method including both CAD systems and fractals. The results of the tests have been represented in section 4 and finally, this paper ends with the conclusions in section 5 .

\section{Literature ReView}

For lung cancer screening, Hidetaka Arimura et al (2004) and Heidi Roberts et al (2005) used computerized scheme and computeraided detection (CAD) to detect automatically lung nodules in lowdose computed tomography images. The first study was based on the subtraction of the image to improve the detection of lung nodules and to eliminate the majority of normal background structures [9]. Using low-dose CT scans in the program of lung cancer screening, 
Samuel Armato et al (2004) studied the detection of lung nodules. In this method, the detection of lung nodules was done automatically in two phases: gray-level thresholding for the early detection of nodule candidates, followed by the application of a rule-based classifier and linear discriminant analysis to distinguish between candidates that correspond to the actual lung nodules and those that correspond to non-nodules [10].

Using PET scan, Wardwell Jr. et al (2005) tried to detect lung nodules. PET scans attempt to diagnose cancer based on glucose metabolism by measuring the absorption of $18 \mathrm{FFDG}$ and provide biochemical information about the lesions in the image [2]. To detect and classify lung cancer in the chest radiography, JunjiShiraishiet al (2006) used computer-aided diagnosis. By using a database of the chest images, this method combined the independent CAD scheme for detection and the classification of lung nodules as a new CAD scheme which can improve the accuracy of radiologists in the detection of lung cancer [11]. Using fractal-feature distance in digital chest radiography to detect subtle lung nodules, Kuniharu Imai et al (2008) assessed quantitatively the influence of anatomic noise. They conducted a fractal analysis of the square chest images using the virtual volume method. The fractal-feature distances between the considered and the reference images were calculated using the pseudo-fractal dimension and complexity, and the square images without the simulated nodule were employed as the reference images [12].

De Boo et al (2009) detected lung nodules and small tumors in chest radiographs using computer aided diagnosis (CAD). They show that $\mathrm{CAD}$ with its detection sensitivity detects nodules better than a first reader, in other words, it is as a second reader [13].Using a two-step approach for feature selection and classifier ensemble construction, Michael Lee et al (2010) detected pulmonary nodules with the help of the computer. In this method, ensemble classifierswere constructed using RSM or GA-based feature selection and were tested via leaveone-out validation with feature selection and classifier training executed within each iteration. They further tested a two-step approach using a GA ensemble to first assess the relevance of the features, andthen using this information to control feature selection during a subsequent RSM step. The base classification was performed using linear discriminant analysis (LDA) [14].

By ensemble-based computer-aided diagnosis, Hui Chen et al (2010) differentiated lung nodules on CT images. In 2012, they differentiated lung nodes by comparing the performance of artificial neural network and logistic regression model in CT scans. In this study, morphological characteristics (size, margins, and internal characteristics) in the CT images and the patient's age, gender and history of hemoptysis were registered for each nodule, and then main operation was performed based on differentiation [15].

Elisa Ventura et al (2010) detected nodal metastatic disease in patients with non-small cell lung cancer and compared positron emission tomography (PET), contrast-enhanced computed tomography (CT), and combined PET-CT [16].

By means of virtual dual-energy radiography, Sheng Chen, Kenji Suzuki (2013) detected lung nodules in a computerized system. They aimed to develop a CADe scheme with improved sensitivity and specificity by the use of virtual dual-energy (VDE) CXRs where ribs and clavicles are suppressed with massive-training artificial neural networks (MTANNs) [17]. Christe et al (2013) used different computeraided detection software (CAD) at different dose levels to detect lung nodules in lung cancer screening. In this method, anthropomorphic lung phantom and artificial lung nodules were used and studied to simulate screening CT-examination at standard dose and 8 different low dose levels. Two radiologists and 3 different computer aided detection software (CAD) were paired to find the highest sensitivity [18]. Qingzhu Wang et al (2013) detected lung nodules using computer- aided detection by SVM based on 3D matrix patterns. By the proposed support vector machine based on three dimensional matrix patterns (SVM3Dmatrix) which improves the classifier of SVM, 3D volume of interest of suspected lung nodules can be used directly as the training samples [19].

\section{Methodology}

\section{A. CAD System}

There are two main computing systems to help radiologists; they are CADe (computer-aided detection system) and CADx (computeraided diagnosis system). CADe systems detect lesions through medical images, while CADx systems aim to measure the characteristics of the lesion. CADe systems have the following objectives [20]:

- Improvement of accuracy in detection.

- Cooperation in early detection of cancer.

- Reduction of the time spent by radiologists in evaluating the test.

CADe systems for the diagnosis of lung nodules are usually composed of five subsystems: acquisition (acquiring medical images), pre-processing (with the aim of improving the quality of the images to increase accuracy), segmentation, nodule detection and elimination of false positive.

$\mathrm{CAD}$ techniques are used to increase productivity and to observe the effect of methods that help the radiologist to detect suspected cases in CT scan by a computerized system as a second reader. The next aim of using CAD is the extraction and analysis of the characteristics of benign or malignant lesions to help radiologists to reduce the number of FP malignant cancer diagnoses. In summary, it can be said that computers play three roles: detection, diagnosis and noise cancellation [21]. Fig. 1 shows the general principles of computer-aided detection systems.

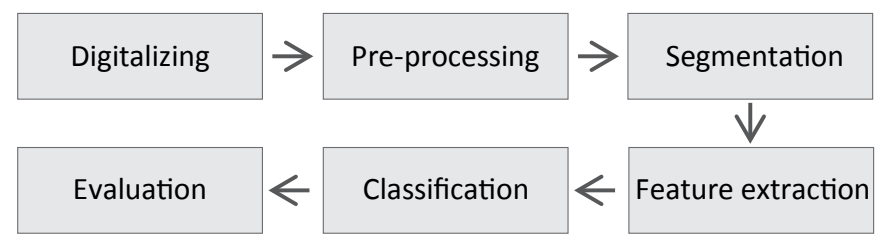

Fig. 1. General principles of computer-aided detection system (CAD)

\section{B. Fractal}

Fractal is a geometric structure consisting of components, in which the same initial structure is obtained by enlarging each component with a certain proportion. In other words, a fractal is a structure in which each component is similar to the whole [22]. There are many methods to calculate the fractal dimensions and each of these methods has its own theoretical basis. Although the algorithms used to calculate the fractal dimensions are different from each other, but they follow the same bases that are briefly described in three stages:

- Quantification of objects using different sizes of steps and stages

- Quantities measured against the size of steps and adjustment of least squares regression line through the data points

- Estimation of fractal dimension as the slope of the regression line

The methods for calculating the fractal dimensions are: box counting method (box- counting dimension), difference box-counting method (DBCM), extended configuration management (XCM), correlation dimension, generalized box-counting dimension and its relationship with the generalized correlation, fractional Brownian motion (FBM), the methods of calculating perimeter.

C. Box Counting Method (Box-Counting Dimension) 
The box counting method has been used in the present paper for calculating the fractal dimension. In this method, signals are provided on limited-scale grids and the effects of grid with the fractal dimensions interact with each other. This method was used for the first time in 1985 by Kolmogorov in the field of dynamic systems. Fig. 2 shows a sample of the implementation of the box-counting method; empty boxes are not counted. The calculation method is so that a box of radius $\mathrm{R}$ is considered and the minimum number of boxes that are required to cover all parts of the geometric object, N (R), is counted. In the study of dynamic systems, the studied set is commonly a set of points in the state-space of the dynamic system. In this case, the empty boxes should not be included in the calculation. The amount of $N(R)$ will be increased with the decrease of R.Box-counting dimension, $D_{b}$, is defined by a number which applies in equation 1 :

$$
\mathrm{N}(\mathrm{R})=\lim _{\mathrm{R} \rightarrow 0} K R^{-D_{b}}
$$

Where $\mathrm{K}$ is the proportionality constant. In practice, to find the amount of the dimension by taking the algorithm on both sides of the above mentioned equation, before taking the limit, the equation 2 is obtained:

$$
D_{b}=\lim _{R \rightarrow 0}\left\{\frac{-\log (N(R))}{\log (R)}+\frac{\log (K)}{\log (R)}\right\}
$$

And by taking the limit on the above equation, the amount of boxcounting dimension is obtained from the equation 3

$$
D_{b}=\frac{\log (\mathrm{N}(\mathrm{R}))}{\log (R)}
$$

The chart of $\log (\mathrm{N}(\mathrm{R}))$ in terms of $\log (\mathrm{R})$ is plotted and in the region where linear behavior can be seen, the slope of the line is calculated and Minkowski dimension is obtained. The topological dimension is always less than or equal to the box-counting dimension and in general the following relationship is available [23].

$$
D_{b} \geq D_{F}
$$

That, $\mathrm{D}_{\mathrm{F}}$ is fractal dimension.

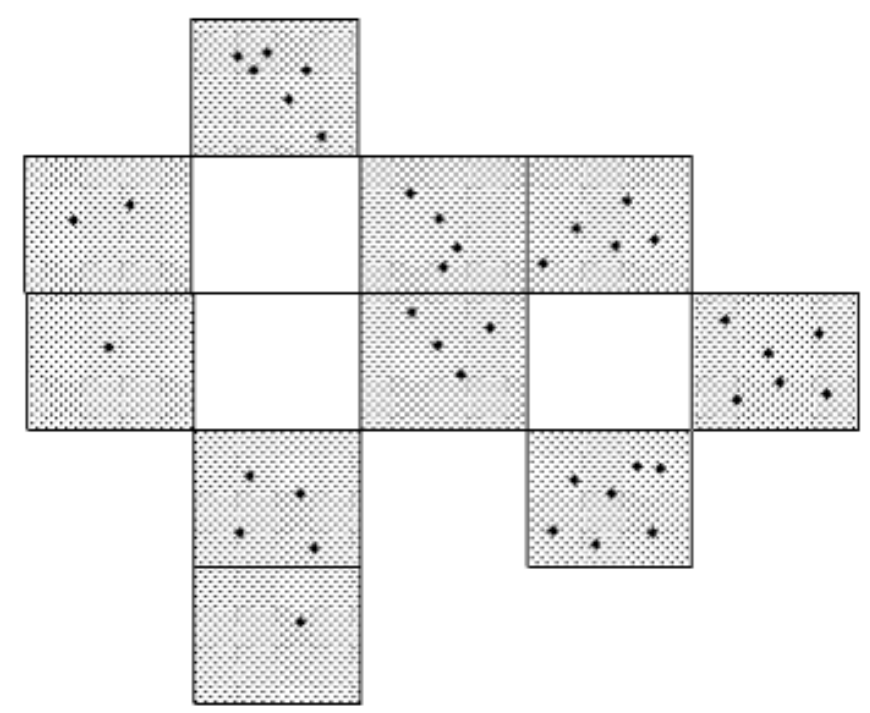

Fig.2. A sample of the implementation of the box-counting method; empty boxes are not counted.

\section{EXPERIMENTAL RESUlts}

In this method, our idea is the use of image analysis and fractal dimension and their combination to detect benign and malignant tumors in lung CT scan images with high contrast, better resolution and less rate of error than other methods.

The proposed method has been tested by using the images obtained from the database of the community of cancer images analysis. 43 images of lung CT scan with the sizes of $128 \times 128$ and the depth of 24 were selected, of which 29 cases were malignant and 14 cases were benign. In other words, the level of gray ranged from 0 (black) to 255 (white). The images were read by an operator of CT scan images and the existing abnormalities were identified and marked. Each image consisted of a set of information: the type and location of anomalies. We selected people who were suffering from the cancer tumor. The images were divided into two groups of benign and malignant. The separation of the images was done based on the Help file in the given web site. To better detect, only a portion of the images which involved regions with cancer tumor were examined. All images were shown with maximum improved contrast so that the full normalized range was from 0 (black) to 1 (white). Fig. 3 shows block diagram of the proposed method.

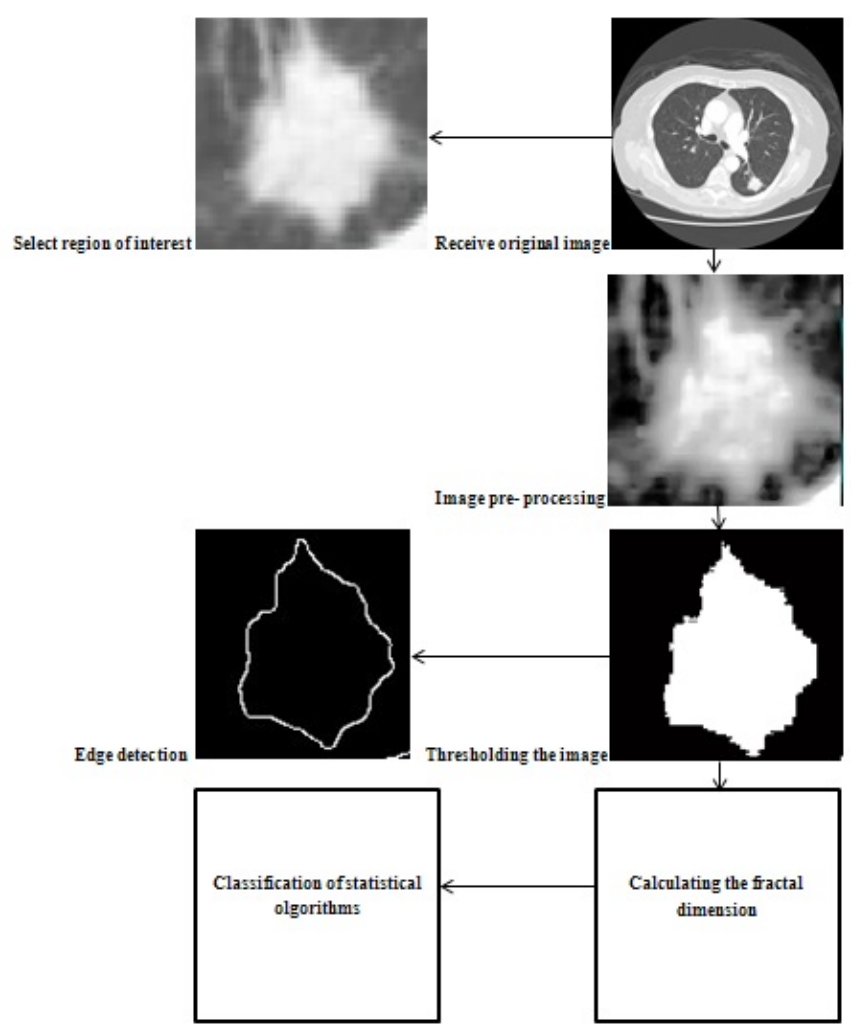

Fig.3. Block diagram of the proposed method.

In the first stage (the selection of the interested area), the regions where were suspected to have cancer tumor were cut from the input images. This process is done manually and the coordinates of the cut location were stored. To pre-process the image, the contrast of the cut images was improved by converting the values in the image intensity using the histogram equalization to increase the accuracy of the tumor diagnosis. Then, the image noise was reduced by the use of the median filtering. The median filter aims to reduce noise and maintain the edge simultaneously. Then segmentation was used to specify a particular area of the image. Segmentation of an image indicates segregation of the image into areas so that the pixels of each area share in a special feature (that can belong to an object). The easiest method for segmenting of an 
image is the thresholding. Thresholding was performed by the use of FCM method which is the fuzzy c-means clustering. FCMTHRESH thresholding was done by 3-class fuzzy c-means clustering. Then edge detection was carried out in MATLAB using the edge function; this function performs the thresholding operation by the partial derivation of the adjacent pixel data and the points in which derivation is maximal are known as edge. In this method, the pixels are searched that are specific to the edge of the object and edge detection is performed in the Fig. using the filters. There are many filters for edge detection. The filter of Canny edge detector has been used in this method. Then, the fractal dimension was calculated for images by the use of box-counting dimension that is show in Fig. 4.

\begin{tabular}{|c|c|c|c|c|}
\hline $\begin{array}{c}\mathrm{Dmm}=25223 \\
\mathbf{0}\end{array}$ & $\begin{array}{c}\mathrm{Dm}=28922 \\
\mathbf{1}\end{array}$ & $\begin{array}{c}\text { Dimm-2947 } \\
\mathbb{Z}\end{array}$ & Dim=2 2364 & $\underset{D}{\mathrm{D} m}=25626$ \\
\hline 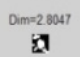 & $\begin{array}{c}\text { Dim=2 } 9621 \\
\$\end{array}$ & Oim=2 20212 & Dime-2553 & Dim=2 26287 \\
\hline Dim=2 7006 & $D^{D i m=27153}$ & Dim-2 2594 & Oime 28712 & $\begin{array}{c}\mathrm{O} m=2 \\
\mathrm{D}\end{array}$ \\
\hline$D^{D}$ & [ & $D$ & [C & Omme2 4904 \\
\hline Dim=2698 & $\begin{array}{l}D i m-27557 \\
\mathbf{d}\end{array}$ & ${ }_{\overline{6}}^{0 . m-2916}$ & Dim-2.5507 & $\mathrm{O}^{\mathrm{m} m \mathrm{~m} 2} 29002$ \\
\hline $\begin{array}{c}\text { Dim-20023 } \\
\mathbb{R}\end{array}$ & ${ }^{D i m-2}=2279$ & D & Dim=2021 & $\mathrm{D}$ imme2 1561 \\
\hline Dim-27105 & ${ }^{D}$ & $D_{m=2} 29006$ & Dimme 0257 & $\mathbf{n}$ \\
\hline D & 13 & tis & a & 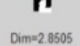 \\
\hline Dim-25005 & Dim-3013 & 口] & 8 & D \\
\hline$D_{i m=22024}$ & $D_{m}=3064$ & $\begin{array}{c}0 \mathrm{~m}=29095 \\
0\end{array}$ & & \\
\hline
\end{tabular}

Fig.4. Calculation of fractal dimension by BoxDim.

The fractal dimension is calculated by coding in MATLAB software. Some examples of output images have been shown in Fig. 5.
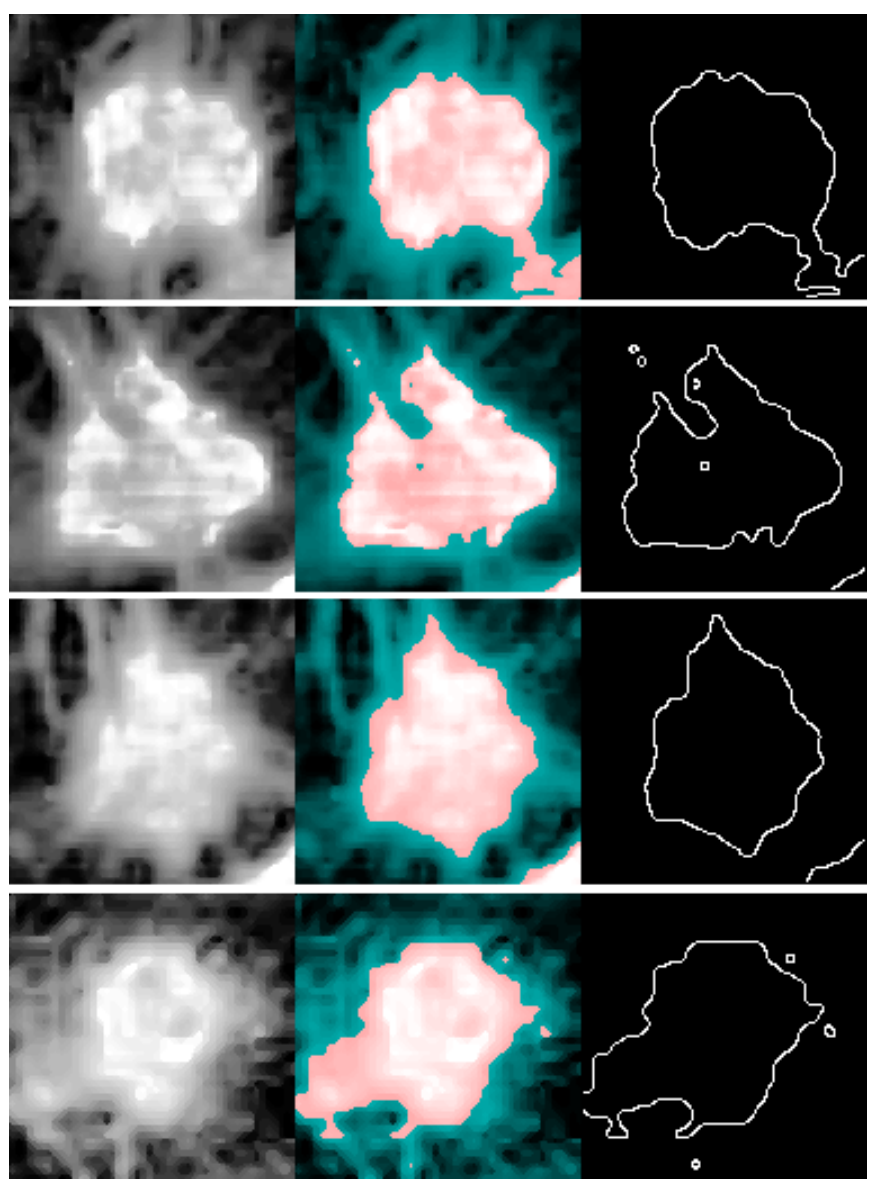

Fig.5. The final results derived from the software for detection of lung nodules based on fractal segmentation.
In this method, box- counting fractal dimension has been calculated. An example of the output data stored in the Data file after deriving MATLAB results has been shown in table 1 .

TABLE I

Final Results of Data

\begin{tabular}{ccc}
\hline \hline Input data No. & $\begin{array}{c}\text { Tumor } \\
\text { (benign: } 1, \text { malignant: } 0)\end{array}$ & BoxDim \\
\hline 18 & 0 & 2.5283 \\
19 & 0 & 2.8921 \\
22 & 0 & 2.9478 \\
24 & 0 & 2.3683 \\
30 & 0 & 2.5626 \\
32 & 0 & 2.8047 \\
34 & 0 & 2.9620 \\
38 & 1 & 2.6211 \\
47 & 0 & 2.8712 \\
48 & 1 & 2.9070 \\
51 & 0 & 2.8712 \\
52 & 1 & 2.8998 \\
54 & 1 & 3.0522 \\
\hline \hline
\end{tabular}

Finally, by the use of Weka software, the results obtained from the fractal dimension were compared with the results of statistical methods such as entropy, mean, standard deviation, Skewness and Kurtosis which have been obtained from coding in MATLAB and applying on input images and then finding output stored in Out Data (in MATLAB). The output of some data stored in Out Data has been shown in table 2. Evaluation has been done based on correlation coefficient (R), mean absolute error (MAE) and root mean square error (RMSE) which has been summarized in table 3 .

TABLE II

Results Saved from MATLAB in Out Data File

\begin{tabular}{cccccccc}
\hline \hline Data No. & Class & BoxDim & Kurtosis & Skewnesss & STD & Average & Entropy \\
\hline 18 & 0 & 2.5283 & 6.4956 & 0.1847 & 24.5 & 132.27 & 6.4550 \\
19 & 0 & 2.8921 & 19.7273 & 0.6678 & 20.41 & 72.4323 & 6.0815 \\
22 & 0 & 2.9478 & 4.4962 & 0.0250 & 24.05 & 99.2346 & 5.9721 \\
24 & 0 & 2.3683 & 1.7701 & 0.0889 & 9.234 & 134.8543 & 6.7318 \\
30 & 0 & 2.5626 & 3.7067 & 0.8463 & 19.70 & 28.8060 & 4.4824 \\
32 & 0 & 2.8047 & 3.5349 & 0.3733 & 11.77 & 99.6131 & 5.4047 \\
34 & 0 & 2.9620 & 2.0430 & -0.5647 & 19.51 & 102.6989 & 5.6951 \\
38 & 1 & 2.6211 & 2.4763 & -0.4398 & 13.76 & 123.7399 & 6.7962 \\
47 & 0 & 2.8712 & 5.3510 & -0.0953 & 14.96 & 91.7998 & 5.6488 \\
48 & 1 & 2.9070 & 3.5091 & 1.2990 & 18.76 & 92.8455 & 6.4508 \\
51 & 0 & 2.8712 & 3.7755 & -0.2651 & 15.17 & 93.7937 & 5.0410 \\
52 & 1 & 2.8998 & 1.7232 & 0.1099 & 19.8 & 142.1170 & 7.0741 \\
54 & 1 & 3.0522 & 6.4988 & -0.2526 & 11.08 & 151.6860 & 7.3466 \\
\hline \hline
\end{tabular}

TABLE III

Comparison of the Results of Statistical Algorithms and Fractals

\begin{tabular}{ccccccc}
\hline \hline $\begin{array}{c}\text { Algorithm } \\
\text { evalution }\end{array}$ & $\begin{array}{c}\text { Box } \\
\text { Dim }\end{array}$ & Kurtosis & Skewness & STD & Average & Entropy \\
\hline R & 0.90 & 0.63 & 0.58 & 0.32 & 0.88 & 0.87 \\
MAE & 0.14 & 3.40 & 0.35 & 3.82 & 5.36 & 0.25 \\
RMSE & 0.17 & 3.92 & 0.46 & 4.61 & 7.21 & 0.30 \\
\hline \hline
\end{tabular}


As can be seen in table 3, the results obtained from fractal dimension have better performance and provide more acceptable responses than statistical algorithms. According to this study and the obtained results, the fractal dimension algorithm can be selected as a preferred method.

\section{Conclusions}

Interpretation and analysis of the medical images constitute the important and major part of the detection field of a machine. Computeraided detection systems are used for all cancers, which cannot be detected by radiologists or CT scan operators. In other words, CAD is used to increase productivity and see the impact of the methods that as the second reader by the computerized system help the operators to diagnose cases which are suspected to lung cancer. In short, it can be said computers play three roles in the analysis of lung cancer: detection, diagnosis and noise cancellation. Then there is a tendency for computer-aided detection systems aimed at helping the medical community to achieve high accuracy and efficiency.

The present study aimed to detect lung nodules on medical images via computerized calculations to help pathologists along with other pathologic methods. Several computerized methods for processing the images of lung cancer have been developed to detect the possible existence of tumors. As it can be seen in the present paper, the use of fractal dimension in the classification of cancer tumors has better results than previous methods.

\section{REFERENCES}

[1] Kenji Suzuki, Hiroyuki Abe, Heber MacMahon, KunioDoi, ImageProcessing Technique for Suppressing Ribs in Chest Radiographs by Means of Massive Training Artificial Neural Network )MTANN(.IEEE TRANSACTIONS ON MEDICAL IMAGING, VOL. 25, NO. 4, APRIL 2006.

[2] Noel R. Wardwell Jr, Pierre P. Massion. Novel Strategies for the Early Detection and Prevention of Lung Cancer. National Center for Biotechnology Information 32(3):259-68, (2005).

[3] Luis M. Montuenga, Ruben Pio. Current challenges in lung cancer early detection biomarkers. Eur J Cancer. 2009 Sep;45Suppl 1:377-8. doi: 10.1016/S0959-8049(09)70055-3.

[4] Jingwei Ma, Qishi Fan, Lianhui Wang, NengqinJia, ZhidongGu, Hebai Shen. Synthesis of magnetic and fluorescent bifunctional nanocomposites and their applications in detection of lung cancer cells in humans. Talanta. (2010) Jun 15;81(4-5):1162-9. doi: 10.1016/j.talanta.2010.01.042.

[5] M.D. Luque de Castro, M.A. Ferna'ndez-Peralbo. Analytical methods based on exhaled breath for early detection of lung cancer. Trends in Analytical Chemistry Volume 38, September 2012, Pages 13-20.

[6] Hui Chen, Jing Zhang, Yan Xu, Budong Chen, Kuan Zhang. Performance comparison of artificial neural network and logistic regression model for differentiating lung nodules on CT scans. Expert Systems with Applications, Volume 39, Issue 13, 1 October 2012, Pages 11503-11509.

[7] Shoji Kido, Chikazumi Kuroda, Shinichi Tamura: Quantification of Interstitial Lung Abnormalities with Chest Radiography: Comparison of Radiographic Index and Fractal Dimension. AcadRadiol (1998).

[8] Khan M. Iftekharuddin, Jing Zheng, Mohammad A. Islam, Robert J. Ogg. Fractal-based brain tumor detection in multimodal MRI. Applied Mathematics and Computation, Volume 207, Issue 1, 1 January 2009, Pages 23-41.

[9] Hidetaka Arimura, ShigehikoKatsuragawa, Kenji Suzuki, Feng Li, JunjiShiraishi, Shusuke Sone, KunioDoi. Computerized Scheme for Automated Detection of Lung Nodules in Low-Dose Computed Tomography Images for Lung Cancer Screening. AcadRadiol. 2004 Jun;11(6):617-29.

[10] Samuel G. Armato, Arunabha S. Roy, Heber MacMahon, Feng Li, KunioDoi, Shusuke Sone, Michael B. Altman. Evaluation of Automated Lung Nodule Detection on Low-dose Computed Tomography Scans From a Lung Cancer Screening Program. AcadRadiol. 2005 Mar;12(3):337-46.

[11] JunjiShiraishi, Hiroyuki Abe, PhD, Feng Li, Roger Engelmann, Heber
MacMahon, KunioDoi: Computer-aided Diagnosis for the Detection and Classification of Lung Cancers on Chest Radiographs: ROC Analysis of Radiologists' Performance. AcadRadiol. 2006 Aug;13(8):995-1003.

[12] Kuniharu Imai, Mitsuru Ikeda, Yukihiro Enchi, TakanagaNiimi. Quantitative assessment of the influence of anatomic noise on the detection of subtle lung nodule in digital chest radiography using fractalfeature distance, Eur J Radiol. 2008 Nov;68(2):353-7.

[13] D.W. De Boo, M. Prokop, M. Uffmann, B. van Ginneken, C.M. SchaeferProkop. Computer-aided detection [CAD] of lung nodules and small tumours on chest radiographs. Eur J Radiol. 2009 Nov;72(2):218-25. doi: 10.1016/j.ejrad.2009.05.062.

[14] Michael C. Lee, Lilla Boroczky, KivilcimSungur-Stasik, Aaron D. Cann, Alain C. Borczuk,Steven M. Kawut, Charles A. Powell. Computer-aided diagnosis of pulmonary nodules using a two step approach for feature selection and classifier ensemble construction. Artificial Intelligence in Medicine Volume 50, Issue 1, September 2010, Pages 43-53.

[15] Hui Chen, Yan Xu, Yujing, Binrong. Neural Network Ensemble-Based Computer-Aided Diagnosis for Differentiation of Lung Nodules on CT Images. Academic Radiology, Volume 17, Issue 5, May 2010, Pages 595602.

[16] Elisa Ventura, Tina Islam, Michael S. Gee, Umar Mahmood,MartaBraschi, Mukesh G. Harisinghan.: Detection of nodal metastatic disease in patients with non-small cell lung cancer: comparison of positron emission tomography (PET), contrast-enhanced computed tomography (CT), and combined PET-CT. Clinical Imaging, 2010 Jan-Feb;34(1):20-8. doi: 10.1016/j.clinimag.2009.03.012.

[17] Sheng Chen, Kenji Suzuki. Computerized Detection of Lung Nodules by Means of "Virtual Dual-Energy" Radiography. IEEE Trans Biomed Eng. 2013 Feb;60(2):369-78. doi: 10.1109/TBME.2012.2226583.

[18] A. Christe, L. Leidolt, A. Huber, P. Steiger, Z. Szucs-Farkas, J.E. Roos,J.T. Heverhagen, L. Ebner. Lung cancer screening with CT: Evaluation of radiologistsanddifferent computer assisted detection software (CAD) as first andsecond readers for lung nodule detection at different dose levels. European Journal of Radiology, Volume 82, Issue 12, December 2013, Pages e873-e878.

[19] Qingzhu Wang, Wenwei Kang, Chunming Wu, Bin Wang. Computeraided detection of lung nodules by SVM based on 3D matrix patterns. Clin Imaging. 2013 Jan-Feb;37(1):62-9. doi: 10.1016/j.clinimag.2012.02.003.

[20] Qiang Li. Recent progress in computer-aided diagnosis oflung nodules on thin-section CT. Comput Med Imaging Graph. 2007 JunJul;31(4-5):248-57.

[21] Allahverdi.F. Analysis and Control of Operating System Error of mammography machine, masterss thesis, Electrical Engineering, Tehran Azad University, Science and Research branch, 2007.

[22] H. Von Koch, «Sur unecourbe continue sans tangente, obtenue par une construction géométriqueélémentaire,» ArkivförMatematik, vol. 1, pp. 681-704, 1904.

[23] M. Schroeder. Fractals, Chaos, Power Laws. Minutes from an Infinite Paradis. W. H. FreemanAnd Company, 1991.

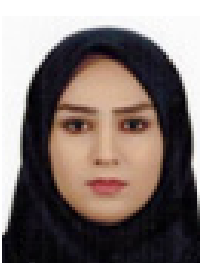

Afsaneh Abdollahzadehrezaie

Afsaneh Abdollahzadehrezaie received the B.S. degree from department of engineering of Islamic Azad University Khoy Branch in 2011. He received the M.Sc. degree in computer science-intelligent system from Islamic Azad University, Khoy Branch, Iran in 2015. His research focuses on medical image processing.

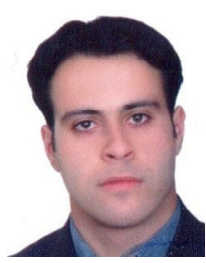

Ali Habiboghli

Ali Habiboghli received the B.S. degree from department of engineering of Islamic Azad University, KHOY Branch in 2004. He received the M.Sc. degrees from electronic, computer engineering and information technology from Islamic Azad University of Qazvin Branch, Iran in 2007. From 2007 to already is with the Islamic Azad University of KHOY Branch. His research focuses on artificial intelligence, algorithms, biometric and image processing. 